\title{
LINC00518 affects the proliferation, invasion and migration of cutaneous malignant melanoma cells via miR-526b-3p/EIF5A2 axis
}

\author{
Jie $\mathrm{Xu}^{1}$, Fan Zhang ${ }^{2}$, Manman Lin ${ }^{1}$ and Yun Tong ${ }^{1 凶}$ \\ IPlastic and Cosmetic Center, Affiliated Jinhua Hospital, Zhejiang University School of Medicine, Jinhua, Zhejiang 321000, P.R. China; \\ 2Department of Dermatology, the First Affiliated Hospital of Wenzhou Medical University, Wenzhou, Zhejiang 325000, P.R. China
}

\begin{abstract}
Objective: To investigate the mechanism of LINC00518 affecting the proliferation, invasion, and migration of cutaneous malignant melanoma (CMM) cells via miR-526b3p/EIF5A2 axis. Methods: qRT-PCR was performed to measure the expression of LINC00518, miR-526b-3p, and EIF5A2 in CMM tissues from 40 patients. Si-LINC00518, pcDNA-LINC00518, miR-526b-3p mimic, miR-526b-3p inhibitor, si-EIF5A2, and their corresponding negative controls were transfected alone or co-transfected into CMM cells A375 and A2058. The expression of LINC00518, miR-526b-3p and EIF5A2 in A375 and A2058 cells was measured. Cell proliferation was tested by CCK-8 assay and EdU assay. Cell invasion and migration were detected by Transwell and scratch tests, respectively. The binding between LINC00518 and miR-526b-3p, and the binding between miR-526b-3p and EIF5A2 were verified by dual-luciferase reporter and RNA pull-down assays. Results: LINC00518 and EIF5A2 were up-regulated and miR-526b-3p was down-regulated in CMM tissues and cells. CMM patients with highly expressed LINC00518 showed decreased survival time than those with lowly expressed LINC00518. Transfection of si-LINC00518, miR-526b-5p mimic or si-EIF5A2 weakened the proliferative, migratory, and invasive abilities of melanoma cells, while transfection of miR-526b-5p inhibitor or pcDNA-LINC00518 enhanced the progression of melanoma cells. Moreover, the proliferative, migratory, and invasive potentials of melanoma cells were decreased after co-transfection of si-EIF5A2 and pCDNA-LINC00518 compared with cells transfected with pCDNA-LINC00518 alone. LINC00518 bound to miR-526b-3p and miR-526b$3 p$ targeted EIF5A2. LINC00518 negatively regulated miR-526b-3p expression but positively regulated EIF5A2. Furthermore, EIF5A2 expression was negatively associated with miR-526b-3p expression. Conclusion: LINC00518 encourages CMM through the miR-526b-3p/EIF5A2 axis in terms of cell proliferation, invasion, and migration.
\end{abstract}

Keywords: cutaneous malignant melanoma, LINC00518, MiR-526b3p, EIF5A2, proliferation, invasion, migration

Received: 17 June, 2021; revised: 14 September, 2021; accepted: 15 September, 2021; available on-line: 21 February, 2022

凶e-mail: tongyun196912@163.com

Abbreviations: CMM, cutaneous malignant melanoma; EIF5A2, cytoplasmic eukaryotic translation initiation factor 5A2; HEMa-LP, Human epidermal melanocytes; GAPDH, glyceraldehyde phosphate dehydrogenase gene; LncRNAs, Long noncoding RNAs

\section{INTRODUCTION}

Melanoma is the leading cause of cancer-related deaths among skin tumors (Liu et al., 2020). The global incidence of cutaneous malignant melanoma (CMM) has continued to increase in spite of the public efforts to promote sun protection behaviors among high-risk groups (Yang et al., 2018). Treatments for melanoma now include surgery, chemotherapy, targeted therapy, and immune therapy (Tsao et al., 2012). Patients with early melanoma have a five-year survival rate of $89 \%$ $96 \%$, compared to $60 \%$ for patients with lymph node metastasis and only $14 \%$ for patients with distant metastases (Donizy et al., 2014; Chen et al., 2019). Melanoma progression presents close associations with multiple aberrantly regulated genes and signaling pathways (Paluncic et al., 2016). Hence it is of great importance to investigate the molecular mechanisms of CMM progression and to find novel biomarkers and pharmaceutical targets for the diagnosis and treatment of CMM.

Long noncoding RNAs (LncRNAs) are defined as transcripts of more than 200 nucleotides not translated into proteins, which are related to cellular mechanisms, gene expression, and pathological conditions (Schmitz et al., 2016, Kopp \& Mendell, 2018). The association between lncRNAs and CMM is gradually established. For example, lncRNA ZFPM2-AS1 deletion was found to restrain the aggressiveness and promote the apoptosis of CMM cells (Liu et al., 2021). Long intergenic non-protein coding RNA 518 (LINC00518) was identified as an independent risk factor in melanoma patients, with up-regulated expression in melanoma tissues (Luan et al., 2019). Nevertheless, the role and function of lncRNA LINC00518 in CMM progression remains to be further elaborated. LncRNAs can act as microRNA (miRNA) sponges to suppress miRNAs, ultimately changing the expression of miRNA-target proteins (Xu et al., 2018; Zhu et al., 2020). MiRNAs, in cancer, acted either as oncogenes or suppressive genes and are rather important for many intracellular pathways involving proliferation and invasion (Lorusso et al., 2020). Previously, miR-526b-3p is lowly expressed in cancers and associated with cancer progression (Liu et al., 2020; Yan et al., 2020, Yin et al., 2020). However, the function of miR-526b-5p in CMM has not been reported. Moreover, cytoplasmic eukaryotic translation initiation factor 5A2 (EIF5A2) expression was upregulated in melanoma and inversely related to patient survival (Khosravi et al., 2016). Notably, miR-526b-5p was predicted by bioinformatics software to have binding sites with both LINC00518 and EIF5A2. 
This study is designed to investigate the function of LINC00518 in CMM progression and to elucidate its potential molecular mechanism involving miR-526b-5p/ EIF52A axis, hoping to discover new insights into diagnostic and prognostic indicators and potential treatment approaches for patients with CMM.

\section{MATERIALS AND METHODS}

\section{Clinical tissue samples}

The malignant melanoma and adjacent normal tissues from 40 melanoma patients in Affiliated Jinhua Hospital, Zhejiang University School of Medicine were collected. None of the patients underwent any radiation treatment or chemotherapy before operation. After surgical resection, the collected tissues were immediately frozen and stored in liquid nitrogen for subsequent RNA or protein extraction. Informed consents were signed by all patients and the study passed the ethical review of Medical Ethics Committee of Affiliated Jinhua Hospital, Zhejiang University School of Medicine.

\section{Cell culture}

Human malignant melanoma cell lines MeWo, CHL-1, A375, and A2058 were all bought from American Type Culture Collection (ATCC), and human epidermal melanocytes HEMa-LP were bought from Invitrogen (Carlsbad, CA, USA). Melanoma cells were pipetted into dulbecco's modified eagle medium (DMEM) (Gibco, Grand Island, NY, USA) containing 10\% fetal bovine serum (FBS), 1\% penicillin, and 1\% streptomycin. Human epidermal melanocytes (HEMa-LP) cells were immersed in medium 254 (Cascade Biologics, USA), and all cells were cultured at $37^{\circ} \mathrm{C}$ using a $5 \% \mathrm{CO}_{2}$ incubator.

\section{Cell transfection}

Si-LINC00518 (2 $\mu \mathrm{g}$, GenePharma, Shanghai, China), LINC00518 overexpression plasmid (pcDNALINC00518, $2 \mu \mathrm{g}$, GenePharma, Shanghai, China), miR526b-3p mimic (50 nM, GenePharma, Shanghai, China), miR-526b-3p inhibitor (50 nM, GenePharma, Shanghai, China), si-EIF5A2 (2 $\mu \mathrm{g}$, GenePharma, Shanghai, China), and their corresponding negative controls (NC) were transfected alone or co-transfected into A375 and A2058 cells. The transfection reagent LipoFiter ${ }^{\mathrm{TM}}$ (Biomart, Shanghai, China) was used for transfection. The operation was performed in accordance with the transfection instructions of LipoFiter ${ }^{\mathrm{TM}}$. The transfection experiment was repeated 3 times and follow-up experiments were conducted $24 \mathrm{~h}$ after transfection.

\section{Quantitative real-time polymerase chain reaction (qRT-PCR)}

TRIZOL (Invitrogen, Carlsbad, CA, USA) was applied to extract total RNA from cells or tissues in each group. After the extraction of total RNA, the concentration and purity of the samples were tested. RNA samples that met the requirements were adjusted to appropriate concentration and reverse transcribed by using random primers as instructed on the reverse transcription kit (TaKaRa, Tokyo, Japan). Gene expression was detected by LightCycler 480 (Roche, Indianapolis, IN, USA) fluorescent quantitative PCR. Reaction conditions were set according to the operating instructions of fluorescent quantitative PCR kit (SYBR Green Mix, Roche
Table 1. Primer sequences

\begin{tabular}{ll}
\hline Name of primer & Sequences \\
\hline U6-F & CTCGCTTCGGCAGCACA \\
\hline U6-R & GCGCTCTTGAGGGAAGCACT \\
\hline Hsa-miR-526b-3p-F & TACGTTCCATAGTCTACCA \\
Gsa-miR-526b-3p-R & TACGCGTAGGGGTTTGACAC \\
GAPDH-F & CCATCTTGGACTTCCAGCCTCC \\
\hline LINC00518-F & CCCTTTGATCCTCAGCGGTCTC \\
LINC00518-R & TATGCAGTGCTCGGCCTTG \\
\hline EIF5A2-F & TTGAACATCCATGTTGTGAGTAGA
\end{tabular}

Note: F, forward; R, reverse; GAPDH, glyceraldehyde-3-phosphate dehydrogenase; LINC00518, long intergenic non-protein coding RNA 518; EIF5A2, eukaryotic initiation factor 5A2.

Diagnostics, Indianapolis, IN), which were specifically as follows: $95^{\circ} \mathrm{C}$ pre-denaturation for $5 \mathrm{~min}, 95^{\circ} \mathrm{C}$ denaturation for $10 \mathrm{~s}, 60^{\circ} \mathrm{C}$ annealing for $10 \mathrm{~s}, 72^{\circ} \mathrm{C}$ extension for $20 \mathrm{~s}$ (in a total of 40 cycles). Each reaction of quantitative PCR was repeated 3 times. U6 was selected to normalize miRNA expression and the glyceraldehyde phosphate dehydrogenase gene (GAPDH) was used to normalize the expression of mRNA and lncRNA. Data analysis was performed by $2^{-\Delta \Delta \mathrm{Ct}}$ method. The amplified primer sequences of each gene and its reference genes are detailed in Table 1.

\section{Western blotting}

RIPA buffer (Beyotime, Shanghai, China) was used to lyse cells and tissues in each group, which were then centrifuged to obtain protein samples. To ensure the same loading quantity of each protein sample, the protein concentration of each sample was determined with GAPDH as the internal control. After the protein concentration was measured with a BCA kit (Beyotime), loading buffer (Beyotime) was added into the corresponding volume of protein and mixed, followed successively by denaturation using a boiling water bath for $3 \mathrm{~min}$. Protein was isolated by using 10\% SDS-PAGE gel. Moreover, the instructions of SDS-PAGE gel preparation kit (Beyotime) were referred to configure 10\% SDS-PAGE gel. Electrophoresis was performed with $80 \mathrm{~V}$ and then performed with $120 \mathrm{~V}$ for $1-2 \mathrm{~h}$ after bromophenol blue entered the separation gel. Membrane transferring was conducted in an ice-bath with a current of $300 \mathrm{~mA}$ for $60 \mathrm{~min}$. After that, the membranes were rinsed in wash buffer for 1-2 min and then were put into blocking buffer for sealing at room temperature for $60 \mathrm{~min}$ or at $4^{\circ} \mathrm{C}$ overnight. At room temperature, the primary antibodies, including rabbit anti-human GAPDH antibody (1:1000, Cell Signaling (5174S), Boston, USA) and rabbit anti-human EIF5A2 antibody (1:1000, Abcam (ab150439), Cambridge, MA, USA), were incubated on a shaking table for $1 \mathrm{~h}$ and washed 3 times with wash buffer, each for $10 \mathrm{~min}$. Then, the membranes were immersed in secondary antibody (horseradish peroxidase conjugated goat anti- 
rabbit IgG, 1:5000, Beijing ComWin Biotech Co., Ltd, Beijng, China) at room temperature for $1 \mathrm{~h}$, and washed 3 times with wash buffer, each for $10 \mathrm{~min}$. Membranes were dripped with developing solution, which then were detected through applying a chemiluminescence imaging system (Bio-Rad, Hercules, CA, USA).

\section{Dual luciferase reporter assay}

The binding sites between miR-526b-3p and LINC00518 were predicted by an online database Jefferson (https://cm.jefferson.edu/rna22/Precomputed/ OptionController? species $=$ HomoSapiens\&type $=\operatorname{lncR}$ NA). The binding sites between miR-526b-3p and EIF5A2 were predicted by an online database TargetScan (http://www.targetscan.org/vert_72/). According to the prediction, the wild-type (WT) and mutated-type (MT) sequences of the binding sites (mut-LINC00518, wt-LINC00518, mut-EIF5A2 and wt-EIF5A2) were designed and synthesized, which were inserted into luciferase reporter vector (pGL3-Basic, Promega, MADISON, WI, USA). The luciferase reporter vector was then co-transfected into HEK293T cells with miR-526b-3p mimic (50 nM, GenePharma) or mimic NC (50 nM, Genepharma), respectively. After transfection, the luciferase intensity in each group was measured using dual-luciferase reporter assay detection kit (Promega, Madison, WI, USA) to determine the binding of miR-526b-3p to LINC00518 and the binding of miR-526b-3p to EIF5A2. The HEK293T cells were accordingly divided into mimic+mut-LINC00518 group, mimic +wt-LINC00518 group, mimic NC+mutLINC00518 group, mimic NC+wt-LINC00518 group, mimic +mut-EIF5A2 group, mimic+wt-EIF5A2 group, mimic $\mathrm{NC}+$ mut-EIF5A2 group, and mimic $\mathrm{NC}+$ wtEIF5A2 group. The experiments were repeated 3 times.

\section{RNA pull-down assay}

Pierce $^{\mathrm{TM}}$ Magnetic RNA-Protein Pull-Down kit (Millipore, Billerica, MA, USA) was used to conduct the RNA pull-down assay. Biotinylated LINC00518, EIF5A2 (Geneseed, Guangzhou, China), and NC were incubated at $25^{\circ} \mathrm{C}$ for $2 \mathrm{~h}$, with A375 and A2058 cell lysates. The LINC00518/miR-526b-3p or EIF5A2/miR-526b-3p complexes were captured using immunomagnetic beads labeled by streptavidin at $25^{\circ} \mathrm{C}$. Then the mixture was incubated with proteinase $\mathrm{K}$-supplemented buffer at $25^{\circ} \mathrm{C}$ for $1 \mathrm{~h}$. The eluted complexes were detected by qRT-PCR.

\section{Cell counting kit- 8 assay (CCK-8 assay)}

With three replicates, cell suspension of the transfected A375 and A2058 cells $(100 \mu \mathrm{l})$ was seeded into 96-well plates. After the cells were incubated in an incubator for $12,24,36$ and $48 \mathrm{~h}, 10 \mu \mathrm{l} \mathrm{CCK}-8$ reagent (Tokyo, Dojindo, Japan) was added into each well. After further incubation, for 1-4 h, the absorbance was measured at $450 \mathrm{~nm}$ wavelength.

\section{EdU assay}

The proliferation of melanoma cells was measured by EdU assay. Twenty-four hours after the transfection of A375 and A2058 cells, the experiment was conducted using an EdU detection kit (RiboBio, Guangzhou, China) following the manufacturer's instructions. Microscopy (Leica DMI3000B) was performed to observe and count positive cells. Moreover, all experiments were repeated 3 times.

\section{Transwell assay}

Matrigel gel activation: the chambers coated with Matrigel gel $\left(-20^{\circ} \mathrm{C}\right)$ were taken out and placed at room temperature. A total of $0.5 \mathrm{~mL}$ serum-free medium was respectively added to a transwell chamber (Coring, New York, USA) and 24-well culture plate. The chamber and plate were placed at $37^{\circ} \mathrm{C}$ and $5 \% \mathrm{CO}_{2}$ for $2 \mathrm{~h}$. Then, the culture medium was removed. Cells in logarithmic growth phase were collected and made into cell suspension which was plated evenly in a six-well plate. The cells were incubated with $5 \% \mathrm{CO}_{2}$ in a $37^{\circ} \mathrm{C}$ incubator, with three replicate wells. When cell fusion reached $70 \% \sim 90 \%$, cells in each group were accordingly treated and incubated in an incubator at $37^{\circ} \mathrm{C}$ and $5 \% \mathrm{CO}_{2}$ for another 24 $\mathrm{h}$. The cells in each group were digested by trypsin, and then were collected and washed twice with PBS. Serum-free DMEM was used to resuspend and adjust cell concentration. Then, the basolateral chamber was added with $600 \mu \mathrm{L}$ medium containing 10\% FBS and the apical chamber was added with $100 \mu \mathrm{l}$ cell suspension, which were incubated for $24 \mathrm{~h}$ in a $37^{\circ} \mathrm{C}$ incubator with $5 \% \mathrm{CO}_{2}$. Thereafter, the apical chamber was taken out to discard the supernatant. Cotton buds were used to wipe off cells that failed to penetrate the membrane inside the chamber. After being fixed with $4 \%$ paraformaldehyde for $20 \mathrm{~min}$, the invasive cells were then stained with Ray-Gimsa dye. High power lens was applied to count and record the number of invasive cells in 5 random fields.

\section{Cell scratch test}

Cells in logarithmic growth phase were collected and made into single cell suspension which was then evenly plated in 6-well plates. After $24 \mathrm{~h}$, cells in each group were treated accordingly and cultured in $5 \% \mathrm{CO}_{2}$ in an incubator at $37^{\circ} \mathrm{C}$. A $100 \mu \mathrm{L}$ sterile pipette tip was used to scratch the cells vertically in 6-well plates to ensure the scratch width in each group is basically the same. The culture medium in the 6-well plates was discarded. Cells were washed twice with PBS and supplemented with serum-free medium for further culture. The $0-\mathrm{h}$ scratch area of cells was observed and photographed as the control. The 6 -well plate was placed in a $5 \% \mathrm{CO}_{2}$ incubator at $37^{\circ} \mathrm{C}$ for another $24 \mathrm{~h}$ of culture and cell migration was observed and photographed.

\section{Statistical analysis}

GraphPad 7.0 software was applied to analyze the data. All data were displayed as mean \pm standard deviation $(\bar{x} \pm s)$. The overall survival rate was analyzed using Kaplan-Meier method and the differences between curves were analyzed using log-rank test. T test was used to compare two groups and one-way analysis of variance (ANOVA) was carried out to compare multiple groups, followed by Tukey's multiple comparisons test. $P<0.05$ was considered to be of statistical significance.

\section{RESULTS}

\section{Up-regulated LINC00518 expression in melanoma tissues is related to adverse clinical outcomes}

The clinical and histological details of melanoma patients enrolled in this study are presented in $\mathrm{Ta}$ ble 2. To understand the function of LINC00518 in 
Table 2. Clinical and histological details of melanoma patients

\begin{tabular}{|c|c|c|c|}
\hline Clinicopathologic features & & Number of patients & Percentage \\
\hline \multirow{2}{*}{ Sex } & Male & 22 & $55 \%$ \\
\hline & Female & 18 & $45 \%$ \\
\hline \multirow{2}{*}{ Age } & $\leq 63$ & 21 & $52.50 \%$ \\
\hline & $>63$ & 19 & $47.50 \%$ \\
\hline \multirow{2}{*}{ Lymph node metastasis } & Yes & 8 & $20 \%$ \\
\hline & No & 32 & $80 \%$ \\
\hline \multirow{2}{*}{ TNM stage } & $|-| I$ & 25 & $62.50 \%$ \\
\hline & III-IV & 15 & $37.50 \%$ \\
\hline \multirow{5}{*}{ Histological subtype } & Superficial spreading melanoma & 11 & $27.50 \%$ \\
\hline & Nodular melanoma & 12 & $30 \%$ \\
\hline & Lentigo maligna melanoma & 3 & $7.50 \%$ \\
\hline & Acral lentiginous melanoma & 13 & $32.50 \%$ \\
\hline & Unknown & 1 & $2.50 \%$ \\
\hline \multirow{4}{*}{ Breslow thickness (mm) } & $\leq 1$ & 2 & $5 \%$ \\
\hline & $1-2$ & 8 & $20 \%$ \\
\hline & $2-4$ & 9 & $22.50 \%$ \\
\hline & $>4$ & 21 & $52.50 \%$ \\
\hline \multirow{2}{*}{ Ulceration of primary tumor } & Yes & 14 & $35 \%$ \\
\hline & No & 26 & $65 \%$ \\
\hline
\end{tabular}
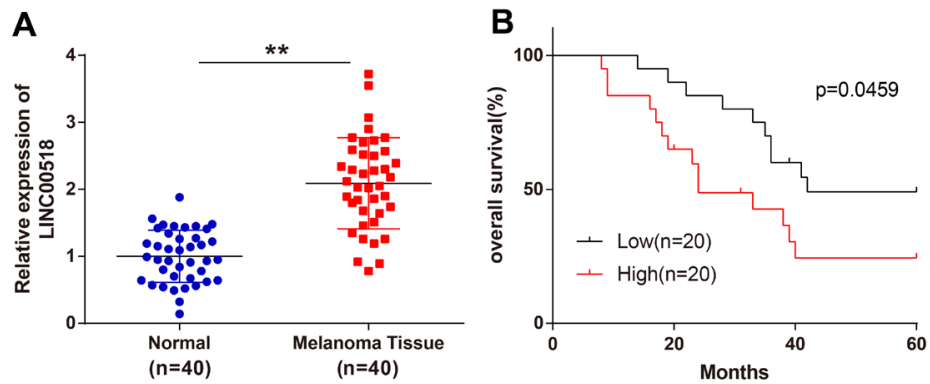

Figure 1. LINC00518 expression in melanoma tissues is up-regulated

Note: (A) qRT-PCR was applied to detect LINC00518 expression in melanoma and their adjacent normal tissues from 40 patients with $\mathrm{CMM},{ }^{* *} P<0.01$, compared to normal group. (B) Kaplan-Meier survival curves, $P=0.0459$, compared to low group.

CMM, qRT-PCR was applied to detect LINC00518 expression in melanoma and adjacent normal tissues of 40 patients. LINC00518 expression in melanoma tissues was significantly higher than that in adjacent normal tissues (Fig. 1A, $P<0.01$ ). Accordingly, 40 patients with melanoma were divided into the lowLINC00518-expression group $(\mathrm{n}=20)$ and the highLINC00518-expression group $(n=20)$ with the median (accurate value: 2.085) of LINC00518 expression in patients with CMM as the cut off value. According to Kaplan-Meier analysis, the overall survival of the lowLINC00518-expression group was longer than that of the high-LINC00518-expression group (Fig. 1B, $P=0.0459)$. These data showed that LINC00518 and melanoma progression were closely related.
Knockdown of LINC00518 inhibits the proliferative, invasive, and migratory behaviors of melanoma cells

LINC00518 expression in MeWo, CHL-1, A375, and A2058 cells was significantly higher than that in HEMa-LP cells (Fig. $2 \AA, P<0.01$ ). After transfection of si-LINC00518, LINC00518 expression in A375 and A2058 cells was significantly decreased (Fig. 2B, $P<0.01)$. CCK-8 and EdU assay results uncovered that the proliferation of A375 and A2058 cells was significantly suppressed after si-LINC00518 transfection (Fig. 2C-E, $P<0.05$ ). Transwell and scratch test results indicated that the invasion and migration of A375 and A2058 cells were significantly reduced after transfection of si-LINC00518 (Fig. 2F-G, $P<0.05$ ). Knockdown of LINC00518 showed suppression of 
A

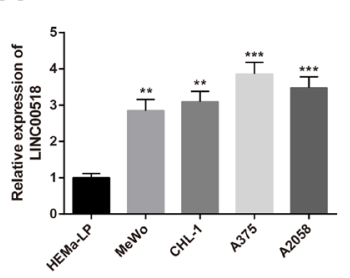

B

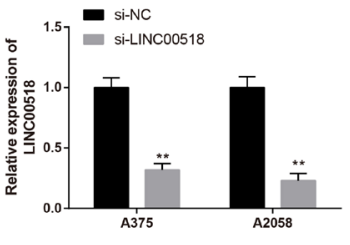

C

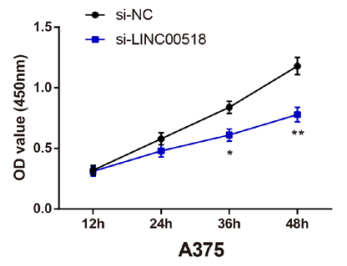

D

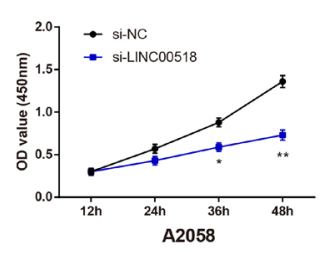

E

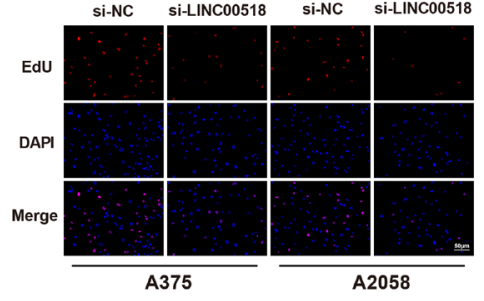

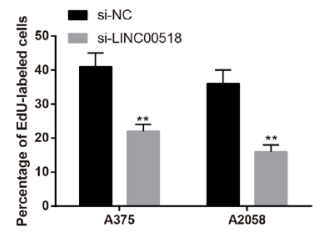

$\mathbf{F}$

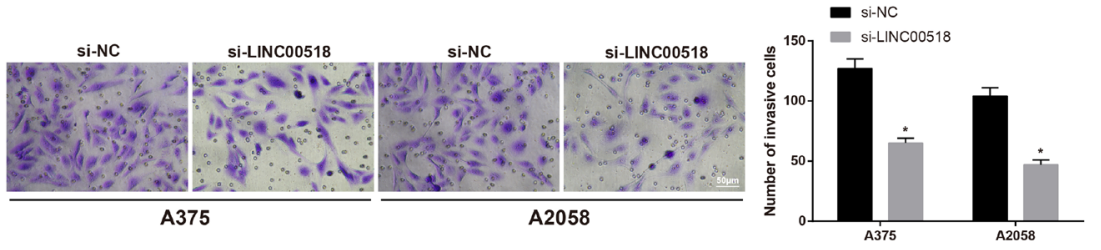

G

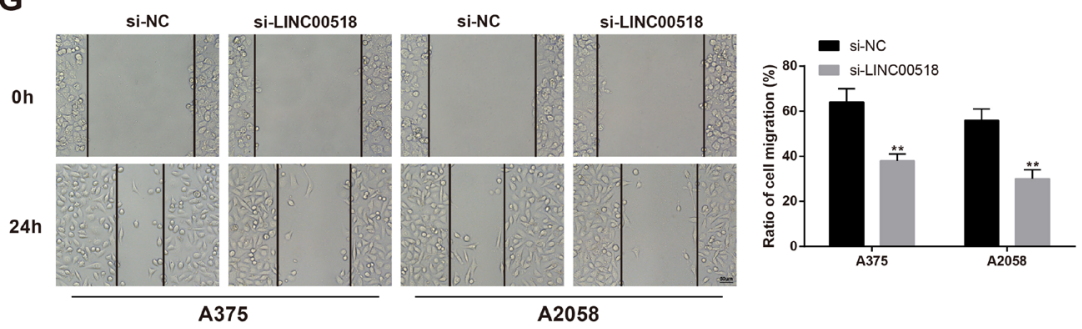

Figure 2. Knockdown of LINC00518 inhibits the proliferation, invasion, and migration of melanoma cells

Note: (A) qRT-PCR was used to detect LINC005418 expression in HEMa-LP, MeWo, CHL-1, A375 and A2058 cells, **P<0.01, ***P<0.001, compared to HEMa-LP group. Si-LINC00518 was transfected into A375 and A2058 cells. (B) qRT-PCR was used to detect LINC00518 expression in A375 and A2058 cells. CCK-8 assay was used to detect OD value of A375 cells (C) and A2058 cells (D) at indicated time. (E) EdU assay was performed to test the proliferation of A375 and A2058 cells. (F) Transwell assay was used to measure the invasion of A375 and A2058 cells. (G) Scratch test was conducted to assess the migration of A375 and A2058 cells. ${ }^{*} P<0.05$, ${ }^{* *} P<0.01$, compared to si-NC group. Data were presented as mean \pm standard deviation $(n=3)$.

the proliferative, invasive, and migratory capacities of melanoma cells.

\section{MiR-526b-3p serves as a target gene of LINC00518}

The binding sites between miR-526b-3p and LINC00518 were predicted by an online database Jefferson. The wild-type (WT) and mutated-type (MT) plasmids containing the 3' UTR region of LINC00518 were constructed for dual-luciferase reporter assay (Fig. 3A). Dual-luciferase reporter assay results showed no significant difference in luciferase activity among mimic+mutLINC00518 group, mimic NC+mut-LINC00518 group, and mimic NC+wt-LINC00518 group. However, the luciferase activity in mimic+wt-LINC00518 group was significantly decreased (Fig. 3B, $P<0.01$ ). RNA pull-down analysis indicated that endogenous miR-526b-3p was dramatically pulled down by biotinylated LINC00518 probe (Fig. 3C-D, $P<0.05$ ). qRT-PCR was applied to detect miR-526b-3p expression in melanoma and their adjacent normal tissues. MiR-526b-3p expression in melanoma tissues was remarkably lower than that in adjacent normal tissues (Fig. 3E, $P<0.01$ ). miR-526b-3p expression in MeWo, CHL-1, A375, and A2058 cells was also distinctly lower than that in HEMa-LP cells (Fig. 3F, $P<0.05$ ). After transfection of si-LINC00518, miR-526b-3p expression in A375 and A2058 cells was significantly enhanced (Fig. 3G, P<0.01). These results uncovered that LINC00518 targeted miR-526b-3p and the expression of the two were negatively associated.

\section{MiR-526b-3p inhibits the proliferative, invasive, and migratory properties of melanoma cells}

To understand the function of miR-526b-3p in the proliferation, invasion, and migration of melanoma cells, miR-526b-3p mimic or miR-526b-3p inhibitor was transfected into A375 and A2058 cells. MiR-526b-3p expression in A375 and A2058 cells was measured by qRTPCR and the results uncovered that miR-526b-3p was 
A

$\begin{array}{ll}\text { wt-LINC00518 } & \text { 5'-GCC--CUGUCUCU--GGUACUUUC-3' } \\ \text { I I I I I I I } \\ \text { miR-526b-3p } & \text { 3'-CGGAGAUUUUCCUUCGUGAAAG-5' } \\ \text { mut-LINC00518 } & \text { 5'-GCC--CUGUCUCU--GGUUGAAAG-3' }\end{array}$

C

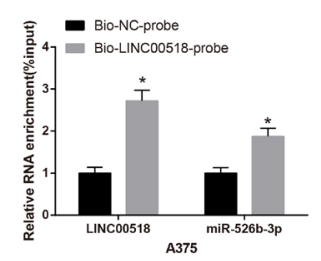

$\mathbf{F}$

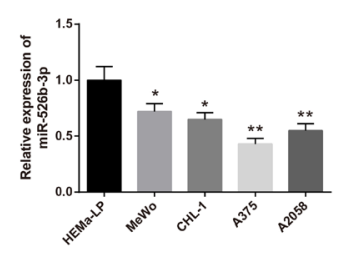

D

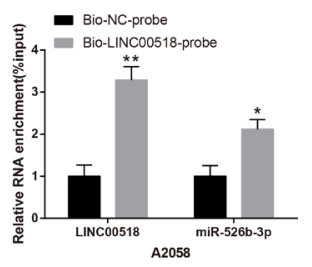

G - sinc

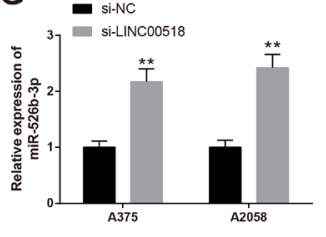

B

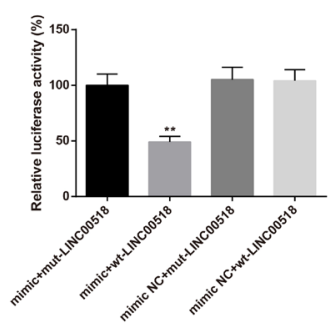

E

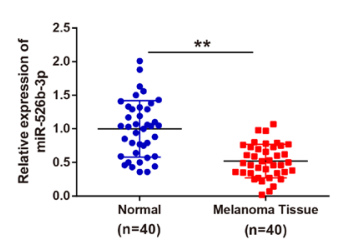

Figure 3. LINC00518 targets miR-526b-3p

Note: (A) The binding sites between miR-526b-3p in the 3' UTR region of LINC00518. (B) The interaction between LINC00518 and miR$526 \mathrm{~b}-3 \mathrm{p}$ was verified by dual-luciferase reporter assay. ${ }^{*} P<0.01$, compared to mimic NC+wt-LINC00518 group. The interaction between LINC00518 and miR-526b-3p in A375 cells (C) and A2058 cells (D) was verified by RNA pull-down assay. ${ }^{*} P<0.05,{ }^{*}{ }^{*} P<0.01$, compared to bio-NC-probe group. (E) The expression of miR-526b-3p in melanoma and their adjacent normal tissues was detected by qRT-PCR. ${ }^{*} P<0.01$, compared to normal group. (F) qRT-PCR was applied to test miR-526b-3p expression in HEMa-LP, MeWo, CHL-1, A375 and A2058 cells. ${ }^{*} P<0.05$, ${ }^{*} P<0.01$, compared to HEMa-LP group. (G) si-LINC00518 was transfected into A375 and A2058 cells and qRT-PCR was used to detect the expression of miR-526b-3p. ${ }^{*} P<0.01$, compared to si-NC group. Data were presented as mean \pm standard deviation $(n=3)$.

overexpressed or knocked down in A375 and A2058 cells (Fig. 4A, P<0.01). According to CCK-8 and EdU assay results, the proliferation of A375 and A2058 cells was markedly restrained after miR-526b-3p mimic was transfected (Fig. 4B-F, $P<0.05$ ), while it was significantly improved after miR-526b-3p inhibitor was transfected (Fig. 4B-F, $P<0.05$ ). Transwell and scratch test results showed that the invasion and migration of A375 and A2058 cells were markedly weakened after transfection of miR-526b-3p mimic (Fig. 4G-K, $P<0.05$ ), while the malignant behaviors of melanoma cells were significantly enhanced after transfection of miR-526b-3p inhibitor (Fig. 4G-K, $P<0.05$ ). All results suggested that miR$526 \mathrm{~b}-3 \mathrm{p}$ inhibited the proliferative, invasive, and migratory properties of melanoma cells.

\section{EIF5A2 expression positively associates with LINC00518 expression, while negatively relates to miR-526b-3p expression}

TargetScan predicted the binding sites between miR526b-3p and EIF5A2, and the wild-type and mutant-type plasmids containing the 3' UTR region of EIF5A2 was constructed for dual-luciferase reporter assay (Fig. 5A). No significant difference in luciferase activity was found among mimic+mut-EIF5A2 group, mimic NC+mut-EIF5A2 group, and mimic NC+wt-EIF5A2 group. However, luciferase activity in mimic+wt-EIF5A2 group was remarkably reduced (Fig. 5B, $P<0.01$ ). RNA pull-down analysis showed that endogenous miR-526b-3p was significantly pulled down by biotinylated EIF5A2 probe (Fig. 5C-D, $P<0.05$ ). qRT-PCR and Western blotting were applied to measure EIF5A2 expression in melanoma and their adjacent normal tissues. It was found that EIF5A2 expression in melanoma tissues was notably higher than that in the adjacent normal tissues (Fig. 5E$\mathrm{F}, P<0.01)$. The mRNA and protein expression of EIF5A2 in MeWo, CHL-1, A375, and A2058 cells was significantly higher than that in HEMa-LP cells (Fig. 5G$\mathrm{H}, \mathrm{P}<0.01)$. EIF5A2 expression was down-regulated after si-LINC00518 transfection in A375 and A2058 cells $(P<0.05)$, while it was up-regulated after miR-526b-3p inhibitor transfection in A375 and A2058 cells $(P<0.01)$. However, EIF5A2 expression was down-regulated in the si-LINC00518+miR-526b-3p inhibitor group compared to that in the miR-526b-3p inhibitor group (Fig. 5I-J, $P<0.05)$. These results suggested that EIF5A2 expression positively associated with LINC00518 expression, while it negatively related with miR-526b-3p expression.

Knockdown of EIF5A2 inhibits the proliferative, invasive, and migratory activities of melanoma cells

Si-EIF5A2 and pcDNA-LINC00518 were transfected alone or co-transfected into A375 and A2058 cells. According to CCK-8 and EdU test results, the proliferation of A375 and A2058 cells was markedly repressed after si-EIF5A2 transfection (Fig. 6A-E, $P<0.05$ ) but 

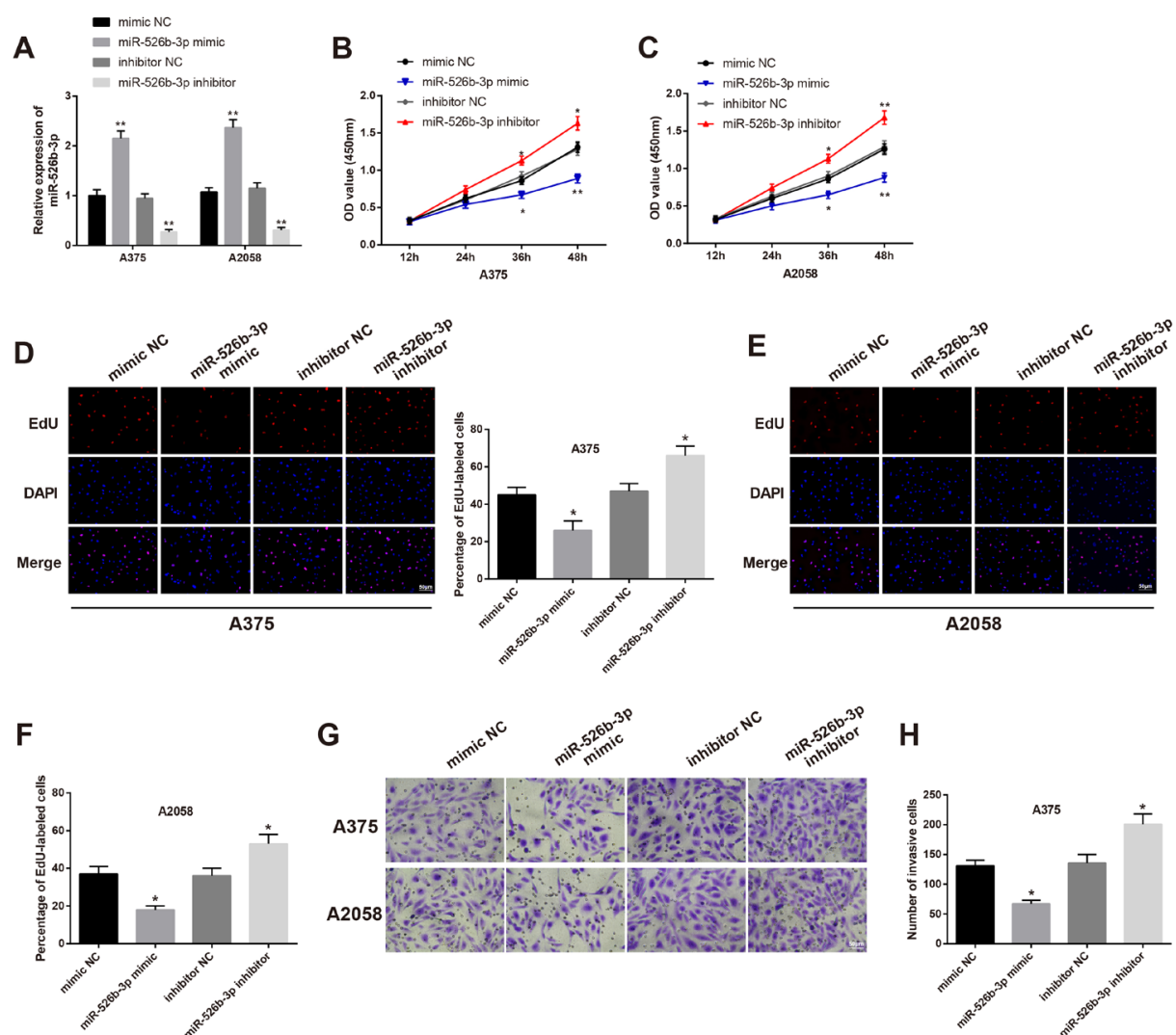

G
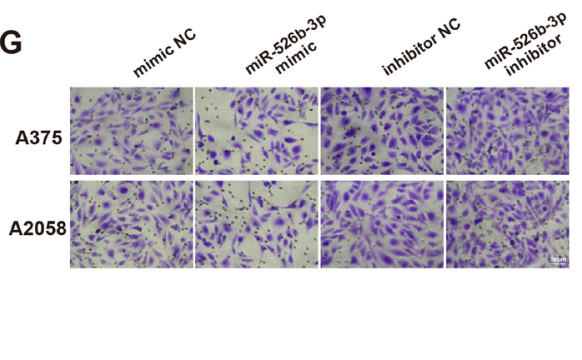

H
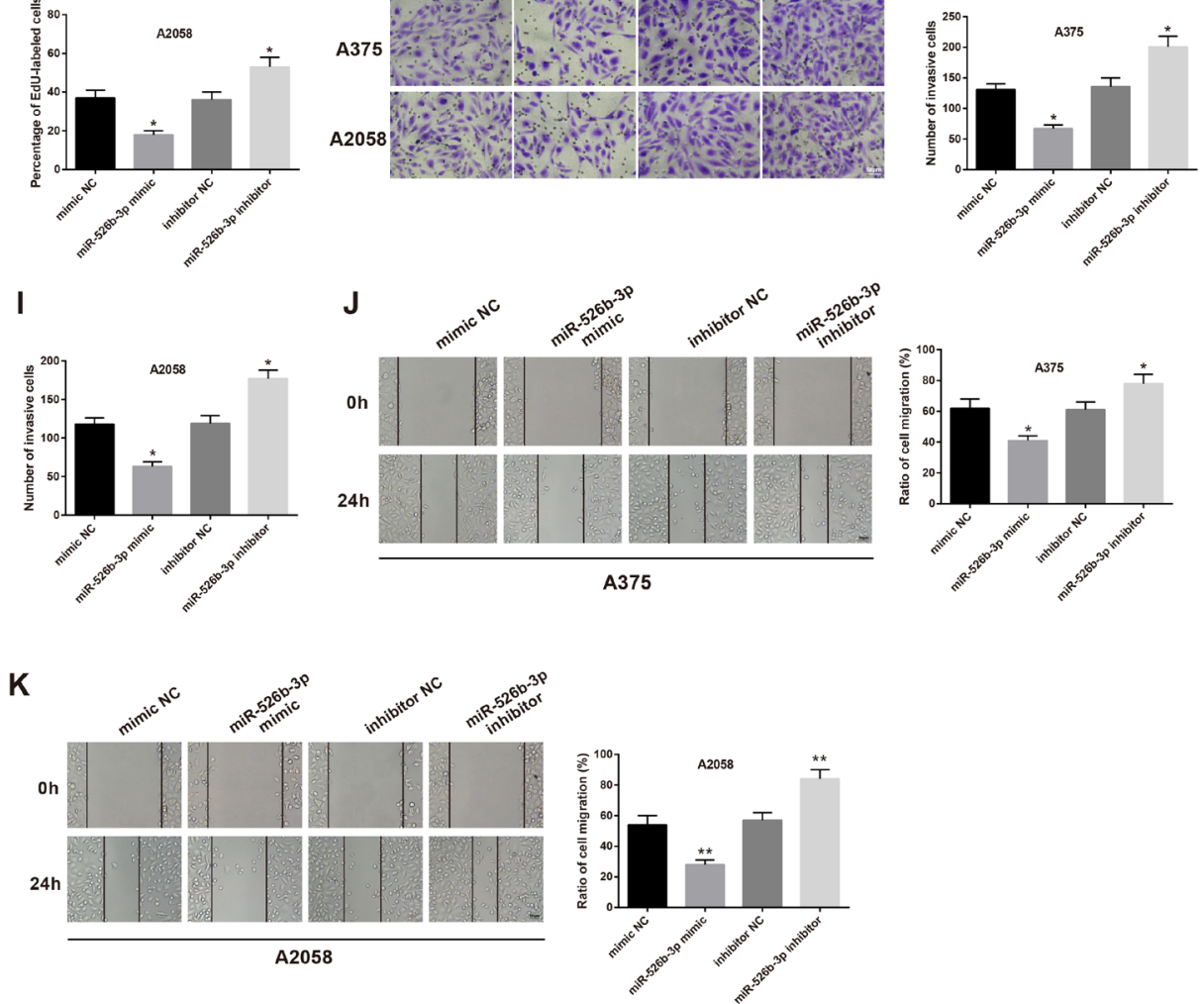

Figure 4. MiR-526b-3p inhibits the proliferation, invasion, and migration of melanoma cells

Note: MiR-526b-3p mimic or miR-526b-3p inhibitor was transfected into A375 and A2058 cells. (A) qRT-PCR was used to detect miR-526b$3 p$ expression in A375 and A2058 cells. CCK-8 assay was performed to assess OD value of A375 (B) and A2058 (C) cells at indicated time. EdU test was applied to measure the proliferation of A375 (D) and A2058 (E-F) cells. (G) The invasion of A375 (H) and A2058 (I) cells was assessed by Transwell assay, and the migration of A375 (J) and A2058 (K) cells was tested by scratch test. ${ }^{*} P<0.05$, ${ }^{* *} P<0.01$, compared to mimic NC group or inhibitor NC group. Data were presented as mean \pm standard deviation $(n=3)$.

it was significantly strengthened after transfection with pcDNA-LINC00518 (Fig. 6A-E, $P<0.05$ ). The proliferation of melanoma cells in the si-EIF5A2+ pcDNALINC00518 group was decreased compared to that in the pcDNA-LINC00518 group (Fig. 6A-E, $P<0.05$ ). Transwell and scratch test results showed that after si-EIF5A2 transfection, the invasion and migration of A375 and A2058 cells were remarkably reduced
(Fig. 6F-J, $P<0.05$ ), while the melanoma cells presented enhanced invasive and migratory abilities after pcDNA-LINC00518 transfection (Fig. 6F-J, $P<0.05$ ). After co-transfection with si-EIF5A2 and pcDNALINC00518, the invasive and migratory abilities of melanoma cells were decreased compared to cells transfected with pcDNA-LINC00518 alone (Fig. 6F-J, $P<0.05$ ). Based on the results, EIF5A2 knockdown showed the 
A

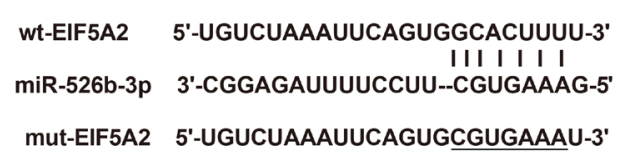

B

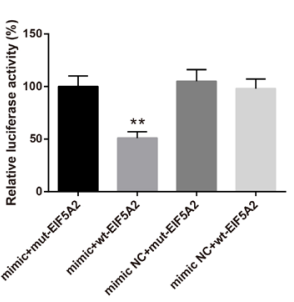

E

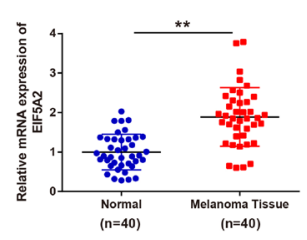

C

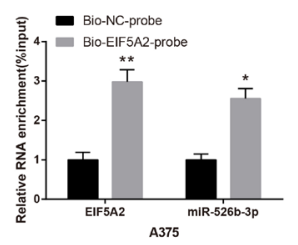

D

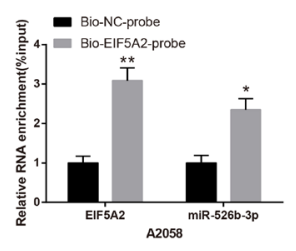

$G$
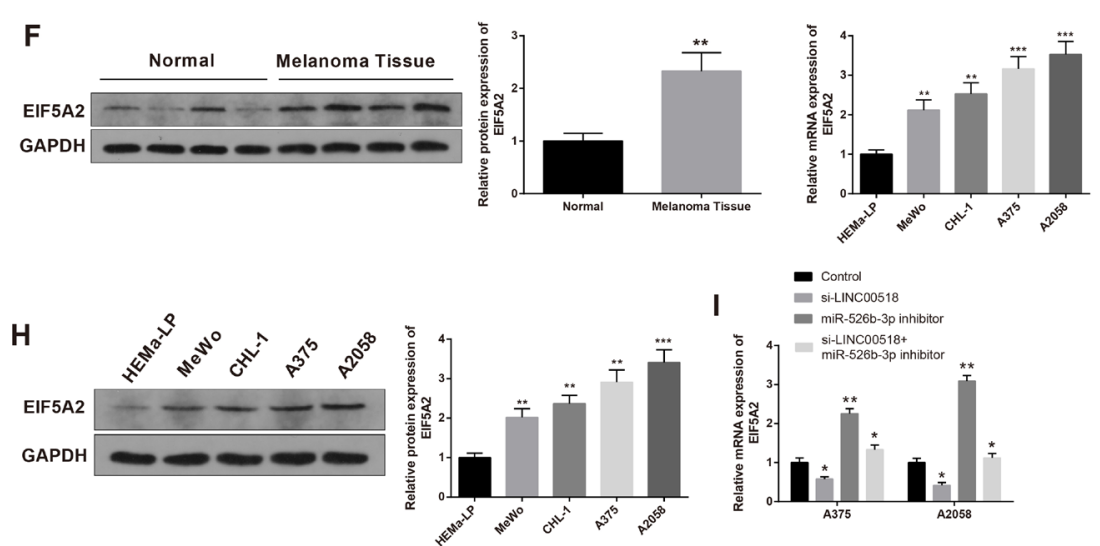

$\mathbf{J}$
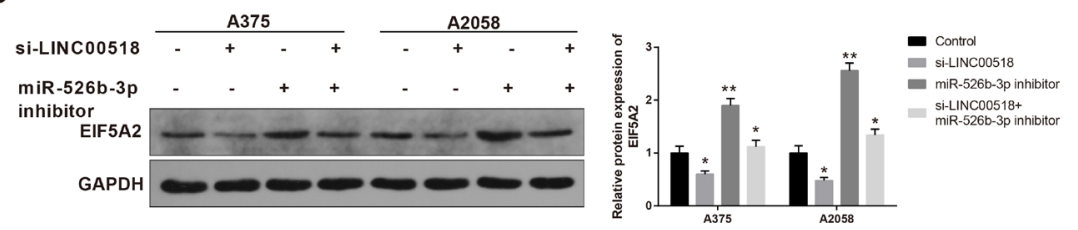

Figure 5. EIF5A2 expression positively correlates with LINC00518 expression, while it negatively correlates with miR-526b-3p expression

Note: (A) The binding sites of miR-526b-3p in the 3'-UTR region of EIF5A2. (B) The interaction between EIF5A2 and miR-526b-3p was verified by dual-luciferase reporter assay. ${ }^{* *} P<0.01$, compared to mimic NC+wt-EIF5A2 group. The interaction between EIF5A2 and miR$526 \mathrm{~b}-3 \mathrm{p}$ in A375 (C) and A2058 (D) cells was verified by RNA pull-down assay. ${ }^{*} P<0.05$, ${ }^{*} P<0.01$, compared to bio-NC-probe group. (E) qRT-PCR and (F) Western blotting were used to detect EIF5A2 expression in melanoma and their adjacent normal tissues. ${ }^{* *} P<0.01$, compared to normal group. (G) GRT-PCR and (H) Western blotting was applied to test EIF5A2 expression in HEMa-LP, MeWo, CHL-1, A375 and A2058 cells. ${ }^{* *} P<0.01,{ }^{* * *} P<0.001$, compared to HEMa-LP group. After si-LINC00518, miR-526b-3p inhibitor or si-LINC00518+miR-526b-3p inhibitor was transfected into A375 and A2058 cells, (I) QRT-PCR and (J) Western blotting were performed to measure EIF5A2 expression. ${ }^{*} P<0.05$, ${ }^{*} P<0.01$, compared to the control group or miR-526b-3p inhibitor group. Data were presented as mean \pm standard deviation $(n=3)$.

inhibitory effect of the proliferative, invasive, and migratory abilities of melanoma cells.

\section{DISCUSSION}

Although early CMM could be simply inspected and easily removed or treated, advanced melanoma still lacks differential diagnosis to other melanocytic lesions, prognostic markers, and efficient treatment (Frohlich, 2010). Dysregulation of lncRNAs is associated with malignant melanoma in terms of tumor growth and metastasis and could be regarded as biomarkers for melanoma (Safa et al., 2020). This study revealed that LINC00518 promot- ed the progression of CMM by targeting the miR-526b3p/EIF5A2 axis.

Numerous studies have indicated that aberrant expression of lncRNA may have an effect on cancer cell proliferation, tumor progression or metastasis (Zhang et al., 2017; Sanchez Calle et al., 2018). Compared with that in adjacent normal tissues, LINC00518 expression was nearly tripled in breast cancer tissues (Chang et al., 2018). Down-regulation of LINC00518 reduced the malignant properties of breast epithelial cancer cells by inhibiting CDX2 methylation through inactivation of Wnt signaling pathway (Wang et al., 2019). In prostate cancer, LINC00518 stimulated paclitaxel resistance by suppressing the expression of miR-216b-5p (He et al., 2019). Liu 


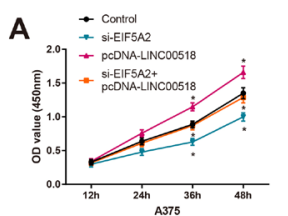

C
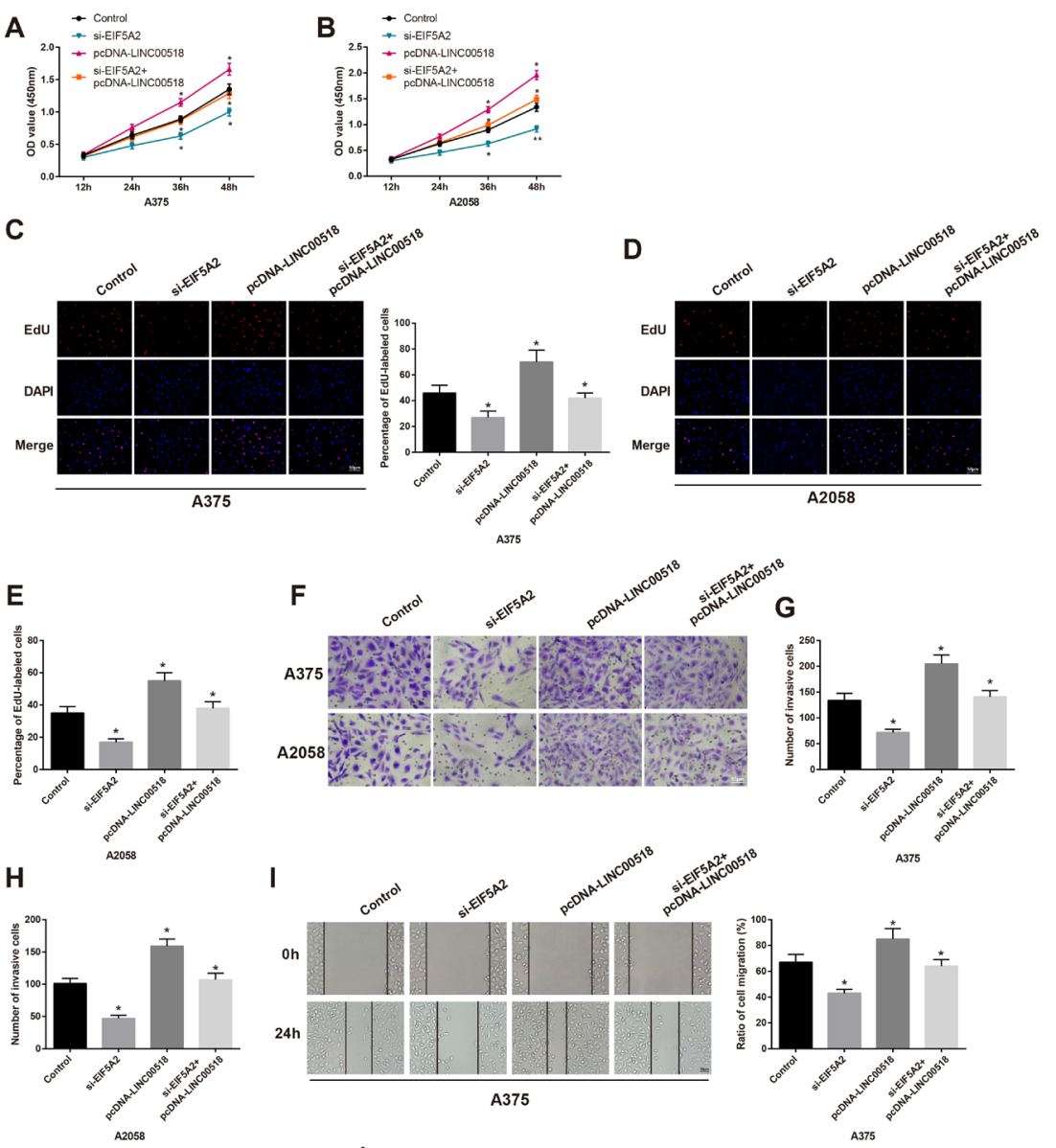

I
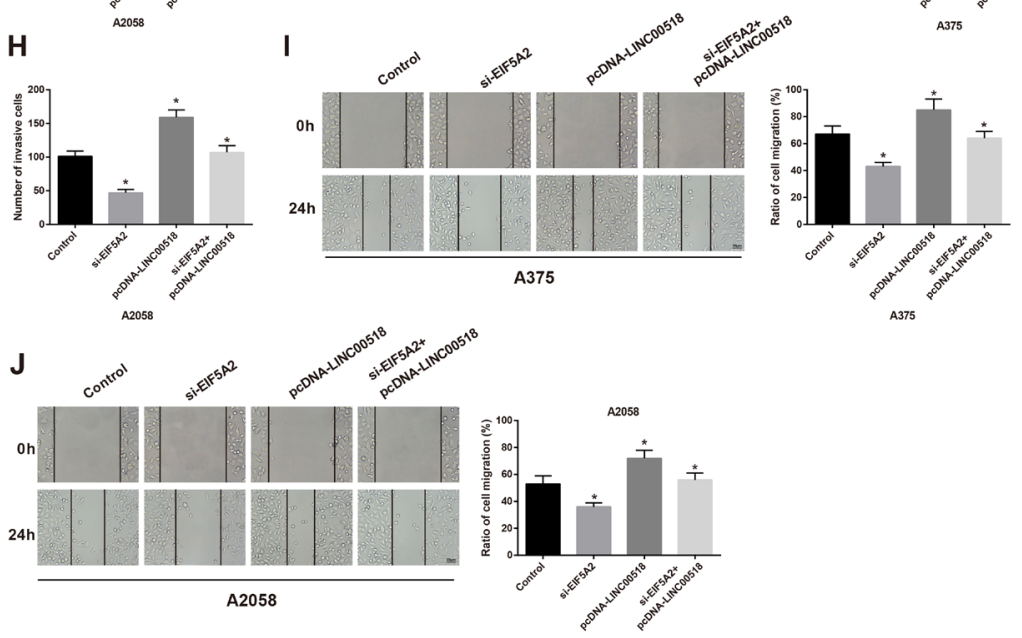

Figure 6. Knockdown of EIF5A2 inhibits the proliferation, invasion, and migration of melanoma cells

Note: After si-EIF5A2 and pcDNA-LINC00518 were transfected alone or co-transfected into A375 and A2058 cells, CCK-8 assay was used to measure OD value of A375 (A) and A2058 (B) cells at indicated time. EdU staining was performed to assess the proliferation of A375 (C) and A2058 (D-E) cells. (F) The invasion of A375 (G) and A2058 (H) cells was detected by transwell assay, and the migration of A375 (I) and A2058 $(\mathbf{J})$ cells were tested by scratch test. ${ }^{*} P<0.05,{ }^{* *} P<0.01$, compared to the control group or pCDNA-LINC00518 group. Data were presented as mean \pm standard deviation $(n=3)$.

et al. reported that LINC00518 sensitized CMM cells to radiotherapy by promoting glycolysis via miR-33a-3p/ HIF-1 $\alpha$ axis (Liu et al., 2021). Moreover, LINC00518 was overexpressed in uveal melanoma and regulated metastatic processes via RNA-RNA interactions (Barbagallo et al., 2020). In this study, high expression of LINC00518 was detected in melanoma tissues. Knockdown of LINC00518 inhibited the proliferation, invasion, and migration of melanoma cells.

As shown by bioinformatics analysis, miR-526b-3p could bind the 3'UTR of LINC00518. Subsequent experiments further verified the targeting relationship between LINC00518 and miR-526b-3p. It is reported that an increase in miR-526b-3p expression suppressed the proliferation, metastasis, and glycolysis of colon cancer cells (Zhang et al., 2016). Down-regulation of miR526b-3p contributed to enhanced proliferation, migra- tion, and invasion of glioma cell lines by increasing the expression of WEE1 (Wu et al., 2019). Introduction of miR-526b-3p inhibited cisplatin resistance in both lung cancer and colorectal cancer (Chen et al., 2021; Zhang et al., 2021). The above evidence demonstrates inhibitory effects of miR-526b-3p on tumor development but the function of miR-526b-3p in CMM has not been reported before. Herein, miR-526b-3p was markedly suppressed in CMM and miR-526b-3p overexpression hindered the proliferation, migration, and invasion of melanoma cells

We further found that EIF5A2 was up-regulated in $\mathrm{CMM}$ and promoted the proliferation and aggressiveness of melanoma cells. EIF5A2 is a phylogenetically conserved gene for eIF5A protein and is often noted for chromosomal instability in cancers (Jenkins et al., 2001). Up-regulation of EIF5A2 has been reported to have 
positive associations with cell proliferation, invasion, and metastasis in cancers such as non-small cell lung cancer, esophageal squamous cell carcinoma, and hepatocellular carcinoma (Chen et al., 2018; Li et al., 2014; Liu et al., 2016). EIF5A2, targeted by phosphorylated Akt, showed significant prognostic value for melanoma and promoted melanoma cell invasion through activation of metalloproteinase-2 (Khosravi et al., 2014). LncRNA LINC00520 enhanced malignancy of melanoma cells and promoted tumor growth and metastasis by increasing the expression of EIF5A2 via miR-125b-5p (Luan et al., 2020). The expression of EIF5A2 was decreased by deoxyhypusine synthase inhibitors that impaired the metastatic potential of melanoma cells (Liu et al., 2021). Consistently, our findings showed that EIF5A2 was highly expressed in melanoma and that knockdown of EIF5A2 displayed suppressive effects on the proliferative, invasive, and migratory abilities of melanoma cells. More importantly, EIF5A2 was identified as a downstream target of miR526b-3p in this study. Knockdown of EIF5A2 reversed the stimulatory effects of LINC00518 overexpression on melanoma cells.

\section{CONCLUSION}

In conclusion, all the results provided evidence that LINC00518 promoted CMM through competitively binding miR-526b-3p to facilitate the expression of EIF5A2. This study of LINC00518/miR-526b-3p/EIF5A2 axis might contribute to our understanding of the pathogenesis of CMM, identification of potential prognostic markers, and discovery of novel therapeutic strategies for clinical CMM treatment. This study has to be seen in light of some limitations despite the findings of the effect of LINC00518 on CMM progression. The functions of LINC00518/miR-526b-3p/EIF5A2 axis in CMM were mainly identified in cellular background. Elaborating works are required to confirm the findings of this study in animal models of CMM. The predictive value of LINC00518 in the diagnosis and therapeutic outcomes of CMM should be further exploited to translate the insight gained from this work into clinical application.

\section{Acknowledgement}

Thanks for all the contributors and participants.

\section{Conflict of interests}

The authors declare that there is no conflict of interests.

\section{Funding}

Not applicable.

\section{REFERENCES}

Barbagallo C, Caltabiano R, Broggi G, Russo A, Puzzo L, Avitabile T, Longo A, Reibaldi M, Barbagallo D, Di Pietro C, Purrello M, Ragusa M (2020) LncRNA LINC00518 acts as an oncogene in uveal melanoma by regulating an RNA-based network. Cancers (Basel) 12(12). https://doi.org/10.3390/cancers12123867

Chang L, Hu Z, Zhou Z, Zhang H (2018) Linc00518 Contributes to multidrug resistance through regulating the MiR-199a/MRP1 axis in breast cancer. Cell Physiol Biochem 48: 16-28. https://doi. org $/ 10.1159 / 000491659$

Chen C, Zhang B, Wu S, Song Y, Li J (2018) Knockdown of EIF5A2 inhibits the malignant potential of non-small cell lung cancer cells. Oncol Lett 15: 4541-4549. https://doi.org/10.3892/ol.2018.7832

Chen KB, Yang W, Xuan Y, Lin AJ (2021) miR-526b-3p inhibits lung cancer cisplatin-resistance and metastasis by inhibiting STAT3- promoted PD-L1. Cell Death Dis 12: 748. https://doi.org/10.1038/ s41419-021-04033-8

Chen X, Gao J, Yu Y, Zhao Z, Pan Y (2019) LncRNA FOXD3-AS1 promotes proliferation, invasion and migration of cutaneous malignant melanoma via regulating miR-325/MAP3K2. Biomed Pharmacother 120: 109438. https://doi.org/10.1016/j.biopha.2019.109438

Donizy P, Kaczorowski M, Leskiewicz M, Zietek M, Pieniazek M, Kozyra C, Halon A, Matkowski R (2014) Mitotic rate is a more reliable unfavorable prognosticator than ulceration for early cutaneous melanoma: a 5-year survival analysis. Oncol Rep 32: 2735-2743. https://doi.org/10.3892/or.2014.3531

Frohlich E (2010) Proteases in cutaneous malignant melanoma: relevance as biomarker and therapeutic target. Cell Mol Life Sci 67: 3947-3960. https://doi.org/10.1007/s00018-010-0469-5

He J, Sun M, Geng H, Tian S (2019) Long non-coding RNA Linc00518 promotes paclitaxel resistance of the human prostate cancer by sequestering miR-216b-5p. Biol Cell 111: 39-50. https:// doi.org/10.1111/boc.201800054

Jenkins ZA, Haag PG, Johansson HE (2001) Human eIF5A2 on chromosome 3q25-q27 is a phylogenetically conserved vertebrate variant of eukaryotic translation initiation factor $5 \mathrm{~A}$ with tissuespecific expression. Genomics 71: 101-109. https://doi.org/10.1006/ geno.2000.6418

Khosravi S, Martinka M, Zhou Y, Ong CJ (2016) Prognostic significance of the expression of nuclear eukaryotic translation initiation factor 5A2 in human melanoma. Oncol Lett 12: 3089-3100. https:// doi.org/10.3892/ol.2016.5057

Khosravi S, Wong RP, Ardekani GS, Zhang G, Martinka M, Ong CJ, Li G (2014) Role of EIF5A2, a downstream target of Akt, in promoting melanoma cell invasion. Br J Cancer 110: 399-408. https:// doi.org/10.1038/bjc.2013.688

Kopp F, Mendell JT (2018) Functional classification and experimental dissection of long noncoding RNAs. Cell 172: 393-407. https://doi. org/10.1016/j.cell.2018.01.011

Li Y, Fu L, Li JB, Qin Y, Zeng TT, Zhou J, Zeng ZL, Chen J, Cao TT, Ban X, Qian C, Cai Z, Xie D, Huang P, Guan XY (2014) Increased expression of EIF5A2, via hypoxia or gene amplification, contributes to metastasis and angiogenesis of esophageal squamous cell carcinoma. Gastroenterology 146: 1701-1713 e9. https://doi. org/10.1053/j.gastro.2014.02.029

Liu KL, Li XY, Wang DP, Xue WH, Qian XH, Li YH, Lin QQ, Li S, Meng FH (2021) Novel allosteric inhibitors of deoxyhypusine synthase against malignant melanoma: design, synthesis, and biological evaluation. J Med Chem 64: 13356-13372. https://doi.org/10.1021/ acs.jmedchem.1c00582

Liu N, Liu Z, Liu X, Duan X, Huang Y, Jin Z, Niu Y, Zhang L, Chen $\mathrm{H}$ (2020) Identification of an immune-related prognostic signature associated with immune infiltration in melanoma. Front Genet 11: 1002. https://doi.org/10.3389/fgene.2020.01002

Liu RR, Lv YS, Tang YX, Wang YF, Chen XL, Zheng XX, Xie SZ, Cai Y, Yu J, Zhang XN (2016) Eukaryotic translation initiation factor 5 A2 regulates the migration and invasion of hepatocellular carcinoma cells via pathways involving reactive oxygen species. Oncotarget 7: 24348-24360. https://doi.org/10.18632/oncotarget.8324

Liu W, Hu X, Mu X, Tian Q, Gao T, Ge R, Zhang J (2021) ZFPM2AS1 facilitates cell proliferation and migration in cutaneous malignant melanoma through modulating miR-650/NOTCH1 signaling. Dermatol Ther 34: e14751. https://doi.org/10.1111/dth.14751

Liu X, Shen S, Zhu L, Su R, Zheng J, Ruan X, Shao L, Wang D, Yang C, Liu Y (2020) SRSF10 inhibits biogenesis of circ-ATXN1 to regulate glioma angiogenesis via miR-526b-3p/MMP2 pathway. $J$ Exp Clin Cancer Res 39: 121. https://doi.org/10.1186/s13046-02001625-8

Liu Y, He D, Xiao M, Zhu Y, Zhou J, Cao K (2021) Long noncoding RNA LINC00518 induces radioresistance by regulating glycolysis through an miR-33a-3p/HIF-1alpha negative feedback loop in melanoma. Cell Death Dis 12: 245. https://doi.org/10.1038/s41419$021-03523-z$

Lorusso C, De Summa S, Pinto R, Danza K, Tommasi S (2020) miRNAs as key players in the management of cutaneous melanoma. Cells 9(2). https://doi.org/10.3390/cells9020415

Luan W, Ding Y, Ma S, Ruan H, Wang J, Lu F (2019) Long noncoding RNA LINC00518 acts as a competing endogenous RNA to promote the metastasis of malignant melanoma via miR-204-5p/ AP1S2 axis. Cell Death Dis 10: 855. https://doi.org/10.1038/s41419019-2090-3

Luan W, Ding Y, Yuan H, Ma S, Ruan H, Wang J, Lu F, Bu X (2020) Long non-coding RNA LINC00520 promotes the proliferation and metastasis of malignant melanoma by inducing the miR-125b-5p/ EIF5A2 axis. J Exp Clin Cancer Res 39: 96. https://doi.org/10.1186/ s13046-020-01599-7

Paluncic J, Kovacevic Z, Jansson PJ, Kalinowski D, Merlot AM, Huang ML, Lok HC, Sahni S, Lane DJ, Richardson DR (2016) Roads to melanoma: Key pathways and emerging plavers in melanoma progression and oncogenic signaling. Biochim Biophys Acta 1863: 770-784. https://doi.org/10.1016/j.bbamcr.2016.01.025 
Safa A, Gholipour M, Dinger ME, Taheri M, Ghafouri-Fard S (2020) The critical roles of lncRNAs in the pathogenesis of melanoma. Exp Mol Pathol 117: 104558. https://doi.org/10.1016/j. yexmp.2020.104558

Sanchez Calle A, Kawamura Y, Yamamoto Y, Takeshita F, Ochiya T (2018) Emerging roles of long non-coding RNA in cancer. Cancer Sci 109: 2093-2100. https://doi.org/10.1111/cas.13642

Schmitz SU, Grote P, Herrmann BG (2016) Mechanisms of long noncoding RNA function in development and disease. Cell Mol Life Sci 73: 2491-2509. https://doi.org/10.1007/s00018-016-2174-5

Tsao H, Chin L, Garraway LA, Fisher DE (2012) Melanoma: from mutations to medicine. Genes Dev 26: 1131-1155. https://doi. org/10.1101/gad.191999.112

Wang HB, Wei H, Wang JS, Li L, Chen AY, Li ZG (2019) Down-regulated expression of LINC00518 prevents epithelial cell growth and metastasis in breast cancer through the inhibition of CDX2 methylation and the Wnt signaling pathway. Biochim Biophys Acta Mol Basis Dis 1865: 708-723. https://doi.org/10.1016/j.bbadis.2019.01.003

Wu M, Li X, Liu Q, Xie Y, Yuan J, Wanggou S (2019) miR-526b$3 \mathrm{p}$ serves as a prognostic factor and regulates the proliferation, invasion, and migration of glioma through targeting WEE1. Cancer Manag Res 11: 3099-3110. https://doi.org/10.2147/CMAR.S192361

Xu S, Sui J, Yang S, Liu Y, Wang Y, Liang G (2018) Integrative analysis of competing endogenous RNA network focusing on long noncoding RNA associated with progression of cutaneous melanoma. Cancer Med 7: 1019-1029. https://doi.org/10.1002/cam4.1315
Yan F, Ma Y, Liu L, Li L, Deng J, Sun J (2020) Long noncoding RNA HOXD-AS1 promotes the proliferation, migration, and invasion of colorectal cancer via the miR-526b-3p/CCND1 axis. J Surg Res 255: 525-535. https://doi.org/10.1016/j.jss.2020.05.078

Yang K, Fung T'T, Nan H (2018) An epidemiological review of diet and cutaneous malignant melanoma. Cancer Epidemiol Biomarkers Prev 27: 1115-1122. https://doi.org/10.1158/1055-9965.EPI-18-0243

Yin G, Tian P, Buhe A, Yan W, Li T, Sun Z (2020) LncRNA LINC00689 promotes the progression of gastric cancer through upregulation of ADAM9 by sponging miR-526b-3p. Cancer Manag Res 12: 4227-4239. https://doi.org/10.2147/CMAR.S231042

Zhang H, Bai M, Zeng A, Si L, Yu N, Wang X (2017) LncRNA HOXD-AS1 promotes melanoma cell proliferation and invasion by suppressing RUNX3 expression. Am J Cancer Res 7: 2526-2535

Zhang R, Zhao J, Xu J, Wang J, Jia J (2016) miR-526b-3p functions as a tumor suppressor in colon cancer by regulating HIF-1alpha. Am J Transl Res 8: 2783-2789

Zhang W, Wang Z, Cai G, Huang P (2021) Downregulation of Circ_0071589 suppresses cisplatin resistance in colorectal cancer by regulating the MiR-526b-3p/KLF12 axis. Cancer Manag Res 13: 2717-2731. https://doi.org/10.2147/CMAR.S294880

Zhu J, Deng J, Zhang L, Zhao J, Zhou F, Liu N, Cai R, Wu J, Shu B, Qi S (2020) Reconstruction of lncRNA-miRNA-mRNA network based on competitive endogenous RNA reveals functional lncRNAs in skin cutaneous melanoma. BMC Cancer 20: 927. https://doi. org/10.1186/s12885-020-07302-5 